Revista de Matemática: Teoría y Aplicaciones 2005 12(1 \& 2) : 151-156

CIMPA - UCR - CCSS ISSN: 1409-2433

\title{
ESTIMATING PARAMETERS OF GUMBEL DISTRIBUTION USING THE METHODS OF MOMENTS, PROBABILITY WEIGHTED MOMENTS AND MAXIMUM LIKELIHOOD
}

\author{
Smail Mahdi * $\quad$ Myrtene CenaC $^{\dagger}$
}

Received/Recibido: 5 May 2004

\begin{abstract}
We derive here estimators for the parameters of the Gumbel distribution using three estimating methods, namely, the probability weighted moments, the moment and the maximum likelihood methods. Furthermore, we compare the performance of these estimators using simulations. Both integer and non-integer orders are considered in the probability weighted moments method. Overall, the results show that the probability weighted moments method outperforms the other methods in the estimation of both $\alpha$ and $\epsilon$ parameters.
\end{abstract}

Keywords: Gumbel distribution, probability weighted moments method, moment method, maximum likelihood method, simulation.

\section{Resumen}

Derivamos estimadores para los parámetreos de la distribución de Gumbel usando tres métodos, esto es, los momentos ponderados de probabilidad, el momento y la máxima verosimilitud. Además, comparamos el rendimiento de estos estimadores usando simulaciones. Tanto el orden entero como no entero son considerados en el método de momentos de probabilidad ponderado. Los resultados muestran, sobre todo, que el método de momentos de probabilidad ponderada es mejor que los demás en la estimación de los parámetros $\alpha$ y $\beta$.

Palabras clave: Distribución de Gumbel, método de momentos ponderados de probabilidad, método de momentos, método de máxima verosimilitud, simulación.

Mathematics Subject Classification: 62F25, 62F03.

\footnotetext{
${ }^{*}$ Department of Computer Science, Mathematics \& Physics, University of the West Indies, Cave Hill Campus, Barbados. Fax: +(246) 425 1327; E-Mail: smahdi@uwichill.edu.bb

${ }^{\dagger}$ Department of Computer Science, Mathematics \& Physics, University of the West Indies, Cave Hill Campus, Barbados. E-Mail: mcenac20b@hotmail.com.
} 


\section{Introduction}

The two-parameter Gumbel distribution is defined through its probability density function

$$
f(x)=\alpha^{-1} \exp -\frac{(x-\epsilon)}{\alpha} \exp \left[-\exp -\frac{(x-\epsilon)}{\alpha}\right]
$$

for $x \in \mathbb{R}, \alpha>0$ and $\epsilon \in \mathbb{R}$. This distribution is used in many research fields including, among others, life testing and water resource management. We derive below the estimates of $\alpha$ and $\epsilon$ using the methods of moments, probability weighted moments and maximum likelihood, based on a random sample. Furthermore, we compare the performance of these estimators using simulations. We start with the probability weighted moments method. It is worth noting that there is often evidence that the maximum likelihood method does not perform well, especially, in the case of small samples. Therefore, other estimating methods have recently been developed. The probability weighted moments method, strongly advocated in Hosking [3], is among these recent methods. This method constitutes the most serious competitor to the maximumm likelihood estimator, according to Davison and Smith [2]. This method has also the advantage of providing a class of linear L-moments with asymptotic normality, see, Hosking [4].

\section{Probability weighted moments method}

In order to obtain the estimates of $\alpha$ and $\epsilon$ by the method of probability weighted moments, we need first to compute the cumulative function and the corresponding inverse cumulative function, which are respectively obtained as,

$$
F(x)=\exp \left[-\exp -\frac{(x-\epsilon)}{\alpha}\right]
$$

and

$$
x(F)=\epsilon-\alpha\{\ln (-\ln (F)\}
$$

The function $x(F)$ is used for the computation of the rth order probability weighted moment $\beta_{r}$ to obtain

$$
\beta_{r}=\int_{0}^{1} x(F) F^{r} d F=\frac{1}{r+1}[\epsilon+\alpha(\gamma+\ln (r+1))]
$$

where $\gamma$ is the Euler's constant, with approximate value 0.577215 . This result is obtained by using subsequently the change of variables $u=-\ln f$ and $m=(r+1) u$. Using a similar equation for $\beta_{s}$ with $s \neq r$, we get from their ratio, the probability weighted moments estimates for $\alpha$ and $\epsilon$ as follows.

$$
\hat{\alpha}=\frac{(r+1) \hat{\beta}_{r}-(s+1) \hat{\beta}_{s}}{\ln (r+1)-\ln (s+1)}
$$

and

$$
\hat{\epsilon}=(r+1) \hat{\beta_{r}}-\hat{\alpha}[\ln (r+1)+\gamma]
$$


where

$$
\hat{\beta}_{r}=\frac{1}{n} \sum_{i=1}^{n} \frac{\left(\begin{array}{c}
i-1 \\
r
\end{array}\right)}{\left(\begin{array}{c}
n-1 \\
r
\end{array}\right)} x_{(i)}
$$

for $r=1, \cdots, n$. The values $x_{(i)}$ for $i=1, \cdots, n$ stand for the order statistics of $x_{1}, \cdots, x_{n}$. We derive below the maximum likelihood estimates for $\alpha$ and $\epsilon$.

\section{Maximum likelihood method}

The Log-likelihood function based on the random sample $x_{1}, \cdots, x_{n}$ is given by

$$
L(\alpha, \epsilon)=-\sum_{i=1}^{n} \frac{x_{i}-\epsilon}{\alpha}-n \ln \alpha-\sum_{i=1}^{n} \exp -\left[\frac{x_{i}-\epsilon}{\alpha}\right]
$$

which admits the partial derivatives

$$
\frac{\partial \ln L(\alpha, \epsilon)}{\partial \epsilon}=\frac{1}{\alpha}\left[n-\sum_{i=1}^{n} \exp -\left[\frac{x_{i}-\epsilon}{\alpha}\right]\right]
$$

and

$$
\frac{\partial \ln L(\alpha, \epsilon)}{\partial \alpha}=\sum_{i=1}^{n}\left[\frac{x_{i}-\epsilon}{\alpha^{2}}\right]-\frac{n}{\alpha}-\sum_{i=1}^{n}\left[\frac{x_{i}-\epsilon}{\alpha^{2}}\right] \exp -\left[\frac{x_{i}-\epsilon}{\alpha}\right]
$$

for $\alpha \neq 0$. The solving of the system $\frac{\partial \ln L(\alpha, \epsilon)}{\partial \epsilon}=\frac{\partial \ln L(\alpha, \epsilon)}{\partial \alpha}=0$, yields the maximum Likelihood (ML) estimates of $\alpha$ and $\epsilon$ as numerical solutions of the following equations

$$
\epsilon=\alpha\left\{\ln n-\ln \sum_{i=1}^{n} \exp -\left[\frac{x_{i}}{\alpha}\right]\right\}
$$

and

$$
\bar{x}=\alpha+\frac{\sum_{i=1}^{n} x_{i} \exp -\left[\frac{x_{i}}{\alpha}\right]}{\sum_{i=1}^{n} \exp -\left[\frac{x_{i}}{\alpha}\right]} .
$$

The estimate of $\alpha$ is explicitly obtained from equation (12) and the estimate of $\epsilon$ is then implicitly obtained from equation (11) after the substitution of the estimate of $\alpha$.

\section{Method of moments}

The usual moment of order $r$ is obtained as

$$
\mu_{r}=E\left(X^{r}\right)=\sum_{k=0}^{r}\left(\begin{array}{l}
k \\
r
\end{array}\right) \alpha^{r-k} \epsilon^{k} \int_{R} y^{r-k} \exp -[y+\exp (-y)] d y
$$


In the particular cases $r=1$ and $r=2$, we get after computation and simplification

$$
\mu_{1}=\alpha \gamma+\epsilon
$$

and

$$
\mu_{2}=\alpha^{2} J+2 \alpha \epsilon \gamma+\epsilon^{2}
$$

where $\gamma \simeq .577215$ and $J \simeq 1.978$. From the above equation we easily obtain the moment method estimates of $\alpha$ and $\epsilon$ as

$$
\begin{gathered}
\hat{\alpha}=\left[\frac{\overline{x^{2}}-\bar{x}^{2}}{J-\gamma^{2}}\right]^{\frac{1}{2}} \\
\hat{\epsilon}=\bar{x}-\gamma \hat{\alpha}
\end{gathered}
$$

where $\bar{x}$ and $\overline{x^{2}}$ are the empirical moments of order 1 and 2. For the computation of $J$, we needed to develop a special numerical procedure.

\section{Discussion}

The comparability of the three considered methods of estimation (method of moments, maximum Likelihood method and method of probability weighted moments) was explored via simulations involving various sample sizes ranging from 5 to 100 and various values of the parameter space. Integer orders were first used in the probability weighted moments. We present tables illustrating the obtained simulation results for the case of classical twoparameter Gumbel distribution that is $\alpha=1$ and $\epsilon=0$. The conclusions derived from these experiments are as follows. First, with respect to the parameter $\epsilon$, we found that the method of probability-weighted moments outperforms both the maximum likelihood and the moment methods for all sample sizes and that the method of maximum likelihood also outperforms the method of moments for all sample sizes. With respect to the parameter $\alpha$, the methods of maximum likelihood and probability-weighted moments outperform the method of moments for all sample sizes. We also notice that the method of maximum likelihood and method of moments perform similarly for large samples. However, the method of probability-weighted moments significantly outperforms both of the other methods of estimation for small to moderate sample sizes. These results are illustrated in Table 1. We concluded then, that the method of probability- weighted moments generally gives more accurate estimates, followed by the method of maximum likelihood. The method of moments performs less satisfactorily, although in some instances the estimates derived are very similar to those of the method of maximum likelihood. Next, we looked at the performance of the probability weighted moment method, refereed to as, the generalized probability weighted moments method in Rassmussen [6]. Our conclusion is that $\alpha$ and $\epsilon$ estimates derived for both integer and non-integer orders $\mathrm{r}$ and $\mathrm{s}$ are generally accurate as illustrated in Tables 2 and 3 in the case of $n=100$. Note that the performance of the generalized probability weighted moments has recently been investigated in Ashkar and Mahdi [1] and, Mahdi and Ashkar [5] for the estimaton of quantiles of Weibull and Log-logitic distributions. We found here that in some cases the non- integer values provide slightly better estimates and in other cases the estimates are not as accurate as those 
derived from the use of integer orders. Because of the inconsistency of the estimates obtained when non-integer values are used and also because of lack of an analytical rule for determining the best non-integer orders, we recommend that integers are used for both orders $r$ and $s$. Furthermore, it also recommended to use small integer order values to avoid over-weighting unduly large sample observations.

\section{Tables}

The tables bellow illustrate the simulation results.

\begin{tabular}{c|cccccc}
\hline \hline$n$ & ML & ML & MM(1,2) & MM $(1,2)$ & PWM $(0,1)$ & $\operatorname{PWM}(0,1)$ \\
\hline & $\alpha$ & $\epsilon$ & $\alpha$ & $\epsilon$ & $\alpha$ & $\epsilon$ \\
5 & 0.8267 & 0.0837 & 0.7925 & 0.1107 & 0.9752 & 0.0053 \\
10 & 0.9167 & 0.0398 & 0.8954 & 0.0576 & 0.9918 & 0.0019 \\
15 & 0.9457 & 0.0199 & 0.9346 & 0.0319 & 1.0001 & 0.0058 \\
20 & 0.9653 & 0.0171 & 0.9656 & 0.0269 & 1.007 & 0.0024 \\
30 & 0.9805 & 0.0135 & 0.9757 & 0.0201 & 1.0103 & 0.0002 \\
50 & 0.9835 & 0.0069 & 0.9801 & 0.0116 & 1.0020 & 0.0010 \\
100 & 0.9930 & 0.0058 & 0.9905 & 0.0087 & 1.002 & 0.0018 \\
\hline \hline
\end{tabular}

Table 1. Empirical estimates of $\alpha$ and $\epsilon$ obtained by the ML, MM and PWM methods with different sample sizes $n$ for the classical two-parameter distribution with $\alpha=1.0$ and $\epsilon=0$. Pairs $(0,1)$ and $(1,2)$ represent the orders used in the methods.

\begin{tabular}{c|ccccccccc}
\hline \hline$r / s$ & 0 & .25 & .50 & .75 & 1.0 & 1.25 & 1.5 & 1.75 & 2.0 \\
\hline 0 & & 1.0199 & 1.0041 & 1.0024 & 1.0024 & 1.0026 & 1.0026 & 1.0027 & 1.0028 \\
0.25 & 1.0199 & & .9848 & .9908 & .9940 & .9960 & .9971 & .9978 & .9984 \\
0.50 & 1.0041 & .9848 & & .9979 & .9999 & 1.0011 & 1.0015 & 1.0018 & 1.0019 \\
0.75 & 1.0024 & .9908 & .9979 & & 1.0021 & 1.0030 & 1.0029 & 1.0031 & 1.0031 \\
1.0 & 1.0024 & .9940 & .9999 & 1.0021 & & 1.0039 & 1.0034 & 1.0034 & 1.0035 \\
1.25 & 1.0026 & .9960 & 1.0011 & 1.0030 & 1.0039 & & 1.0029 & 1.0031 & 1.0032 \\
1.50 & 1.0026 & .9971 & 1.0015 & 1.0029 & 1.0035 & 1.0029 & & 1.0034 & 1.0034 \\
1.75 & 1.0027 & .9978 & 1.0018 & 1.0031 & 1.0034 & 1.0031 & 1.0034 & & 1.0034 \\
2.0 & 1.0028 & .9984 & 1.0019 & 1.0031 & 1.0035 & 1.0032 & 1.0034 & 1.0035 & \\
\hline \hline
\end{tabular}

Table 2. Generalized Probability weighted moments estimates of $\alpha$ obtained with real orders $r, s=0(.25) 2$ in the case $n=100$. The targeted parameter values are $\alpha=1.0$ and $\epsilon=0$. 


\begin{tabular}{l|ccccccccc}
\hline \hline$r / s$ & 0 & .25 & .50 & .75 & 1.0 & 1.25 & 1.5 & 1.75 & 2.0 \\
\hline 0 & & -.0082 & .0009 & .0019 & .0019 & .0018 & .0017 & .0017 & .0016 \\
0.25 & -.0082 & & .0199 & .0151 & .0125 & .0109 & .0102 & .0094 & .0089 \\
0.50 & .0009 & .0199 & & .0069 & .0050 & .0039 & .0035 & .0032 & .0029 \\
0.75 & .0019 & .0151 & .0069 & & .0022 & .0012 & .0012 & .0011 & .0010 \\
1.0 & .0019 & .0125 & .0050 & .0022 & & -.0001 & .0005 & .0005 & .0005 \\
1.25 & .0018 & .0109 & .0039 & .0012 & -.0001 & & .0013 & .0010 & .0009 \\
1.50 & .0017 & .0101 & .0035 & .0012 & .0005 & .0013 & & .0007 & 0006 \\
1.75 & .0017 & .0094 & .0032 & .0011 & .0005 & .0010 & .0007 & & .0005 \\
2.0 & .0017 & .0089 & .0029 & .0011 & .0005 & .0009 & .0006 & .0005 & \\
\hline \hline
\end{tabular}

Table 3. Generalized Probability weighted moments estimates of $\epsilon$ obtained with real orders $r, s=0(.25) 2$ in the case $n=100$. The targeted parameter values are $\alpha=1.0$ and $\epsilon=0$.

\section{Acknowledgments}

This work is supported in part by UWI research grants and this support is acknowledged and well appreciated.

\section{References}

[1] Ashkar, F.; Mahdi, S. (2003) "Comparison of two fitting methods for the log-logistic distribution", Water Resources Research 39(8).

[2] Davison, A.C.; Smith, R.L. (1990) "Models for exceedances over high threshold", J. R. Stat. Soc. B 52(3): 393-442.

[3] Hosking, J.R.M. (1986) "The theory of probability weighted moments", Research Report RC12210, IBM Thomas J. Watson Research Center, New York.

[4] Hosking, J.R.M. (1990) "L-Moments: analysis and estimation of distributions using linear combinations of order statistics", J. R. Stat. Soc. B 52(1): 105-124.

[5] Mahdi, S.; Ashkar, F. (2004) "Exploring generalized probability weighted moments, generalized moments and maximum likelihood estimating methods in two-parameter Weibull model", Journal of Hydrology 285: 62-75.

[6] Rasmussen, P. (2001) "Generalized probability weighted moments: application to the generalized Pareto distribution", Water Resour. Res. 37(6): 1745-1751. 